\section{Mobilidade educacional intergeracional, discriminação e hipertensão arterial em adultos do Sul do Brasil}

Intergenerational educational mobility, discrimination, and hypertension in adults from Southern Brazil

\section{Movilidad educacional intergeneracional, discriminación e hipertensión arterial en adultos del Sur de Brasil}

Waleska Nishida 1

Emil Kupek 1

Carla Zanelatto 1

João Luiz Bastos 1

doi: 10.1590/0102-311X00026419

\begin{abstract}
A hipertensão arterial sistêmica (HAS) é um relevante problema de saúde pública mundial, marcado por desigualdades sociais. No Brasil, estudos sobre a HAS adotando uma perspectiva teórica de curso de vida são escassos. O presente artigo visa a analisar a relação entre mobilidade educacional intergeracional (MEI) e HAS em adultos brasileiros, verificando o impacto da discriminação interpessoal e da cor/"raça" nesta relação. Foram analisados dados dos pais e de 1.720 adultos, entre 20 e 59 anos, do Estudo EpiFloripa Adulto. Modelos de regressão multinível com efeitos aleatórios foram estimados. Os efeitos fixos mostraram relação inversa entre MEI e odds de HAS, com significância estatística para MEI alta (modelo paterno: $O R$ [odds ratio] =0,39, $p=0,006$; modelo materno: $O R=0,35, p=0,002$; e modelo familiar: $O R=0,35, p=0,001)$. Análises de interação demonstraram, por sua vez, que situações de discriminação podem atuar conjuntamente com a MEI desfavorável, elevando a odds de HAS, especialmente entre negros e pardos. Conclui-se que a MEI constantemente alta é capaz de reduzir significativamente a odds de HAS, mas que a discriminação pode intensificar o efeito de baixos níveis de educação, especialmente em segmentos da população socialmente marginalizados.
\end{abstract}

Iniquidade Social; Doenças Cardiovasculares; Racismo; Mobilidade Social; Análise Multinível

\section{Correspondência}

W. Nishida

Av. Santa Catarina 209, apto. 303, Florianópolis, SC 88070-740, Brasil.

waleska.nis@gmail.com

1 Universidade Federal de Santa Catarina, Florianópolis, Brasil. 


\section{Introdução}

A hipertensão arterial sistêmica (HAS) é uma doença crônica não transmissível, frequentemente assintomática, considerada um dos principais fatores de risco para doenças cardiovasculares. É caracterizada pela mensuração sistemática de níveis pressóricos sistólico/diastólico iguais ou superiores a 140/90mmHg 1 .

A ocorrência da HAS tem sido frequentemente marcada por desigualdades sociais 2,3. Se, por um lado, a prevalência da doença tende a diminuir em muitos países ricos, vem aumentando em países de baixa e média rendas ${ }^{3}$. No Brasil, a prevalência de HAS atinge aproximadamente $30 \%$ da população adulta 4 . Nesse contexto, a ocorrência de HAS tem sido relacionada a diferentes indicadores de posição socioeconômica, tais como ocupação, renda e educação ${ }^{2}$. Entre esses, ressalta-se que a educação tem se mostrado forte e inversamente associada à ocorrência da HAS no Brasil 4,5,6. As evidências mais frequentes dessa associação no país se referem à influência da escolaridade sobre a ocorrência de HAS em estudos transversais 5,6 .

As desigualdades observadas na ocorrência de HAS segundo cor, etnia ou "raça" também têm recebido destaque. Observa-se maior ocorrência da doença entre indivíduos classificados como negros e pardos, quando comparados a brancos 6,7. A literatura tem sugerido que as desigualdades "étnico-raciais" em HAS refletem mais condições sociais desfavoráveis relacionadas à discriminação interpessoal e ao racismo estrutural do que a diferenças biológicas entre brancos e negros 6,8.

Nesse contexto, o presente artigo visa a analisar a relação entre mobilidade educacional intergeracional (MEI) e HAS em adultos brasileiros. Em adição, pretende-se verificar o papel da discriminação interpessoal e da cor da pele como modificadores de efeito da relação entre MEI e HAS. Com isso, pretende-se contribuir para a compreensão das desigualdades em HAS em países de baixa e média rendas e, consequentemente, melhor direcionar políticas públicas que visem a promover a saúde das populações socialmente excluídas ou marginalizadas.

\section{Mobilidade educacional, discriminação e hipertensão arterial no Brasil}

A epidemiologia do curso de vida é o estudo de processos biológicos, comportamentais e psicossociais de longo prazo que vinculam o risco à saúde de adultos a exposições físicas ou sociais que atuam durante a gestação, infância, adolescência, vida precoce ou adulta, ou eventos transmitidos entre gerações 9 .

Destaca-se aqui o modelo de mobilidade social, o qual considera que a saúde dos indivíduos pode ser influenciada pela variação da posição social ao longo do ciclo vital, incluindo ascensão social, mobilidade social descendente, bem como a permanência na mesma posição por períodos indeterminados. Essa variação pode ocorrer tanto dentro de uma mesma geração (intrageracional) como entre gerações diferentes (intergeracional) 10 .

No Brasil, tal como ocorre em outros países de baixa e média rendas, as desigualdades sociais persistem ${ }^{11}$. Análises de mobilidade intergeracional indicam tendência de persistência em determinada posição socioeconômica, especialmente nos extremos sociais baixo e alto ${ }^{12}$. Além disso, mesmo havendo mobilidade ascendente, o "salto" dado pelos menos favorecidos em relação aos seus pais mostra-se insuficiente para garantir igualdade material em relação à parcela da população mais bem provida de recursos 13 .

Vale ressaltar que, apesar da associação inversa entre escolaridade e HAS 5,6, pouco se sabe sobre a relação deste desfecho de saúde com a MEI no Brasil. Apenas um estudo brasileiro foi encontrado, mas este se restringiu à análise da mobilidade social intrageracional e HAS, não tendo sido observada associação estatisticamente significativa 14 .

O comportamento do nível educacional entre gerações no Brasil evidencia também desigualdades relativas à cor, etnia ou "raça”. A probabilidade de negros e pardos permanecerem em níveis baixos de educação é maior do que entre brancos 13,15. Essa desigualdade "racial” em educação reflete, segundo diversos autores, a discriminação interpessoal e o racismo estrutural, os quais se inserem numa trajetória histórica do país baseada na escravidão e nas relações de poder estabelecidas no período colonial brasileiro 16 . 
Considerando que o Brasil é um país de desigualdades sociais intensas e persistentes, com significativa população de negros e pardos, onde a HAS é um problema de saúde pública, produzir mais estudos que explorem a ligação entre a discriminação e as desvantagens socioeconômicas que afetam a pressão arterial (PA) da população são de grande importância.

\section{Métodos}

\section{Participantes}

Foram analisados dados dos 1.720 participantes do Estudo EpiFloripa Adulto (de 20 a 59 anos na linha de base). O estudo iniciou em 2009 com o primeiro seguimento ocorrendo em 2012 ( $\mathrm{n}=1.222$ ) e o terceiro, em 2014 ( $\mathrm{n}=862$ ). Em 2009 e 2012, as entrevistas foram realizadas nos domicílios dos participantes, localizados na área urbana da cidade de Florianópolis, Santa Catarina, Brasil. O processo de amostragem ocorreu por conglomerados em dois estágios, sendo o primeiro referente ao sorteio dos setores censitários classificados por decis de renda do chefe do domicílio, os quais constituíram as unidades primárias de análise; e o segundo estágio, referente aos domicílios a serem visitados. Gestantes com mais de 3 meses e mulheres com recém-nascidos nos últimos seis meses não tiveram aferidas a PA e as medidas antropométricas. Na última onda, os participantes se deslocaram até um centro organizado para a coleta de dados e realização das aferições. Nas três ondas, a PA foi mensurada de duas a três vezes, utilizando-se um esfigmomanômetro digital de pulso. O questionário aplicado em 2009 incluiu questões sobre o nível de educação dos participantes, diagnostico prévio de HAS e uso de medicamento anti-hipertensivo. Na onda de 2012, dados sobre o nível de educação parental foram coletados, além de informações sobre experiências discriminatórias em nível individual. Em 2014, informações sobre o nível educacional dos participantes foram novamente coletadas.

O Comitê de Ética em Pesquisa com Seres Humanos (CEPSH) da Universidade Federal de Santa Catarina aprovou o projeto do estudo sob os protocolos: número 351/08, de 15 dezembro de 2008 e número 1772/11, de 28 de fevereiro de 2011. Os participantes assinaram um termo de consentimento livre e esclarecido após a explicação dos objetivos da pesquisa. O detalhamento dos procedimentos metodológicos na linha de base do Estudo EpiFloripa Adulto foi descrito em publicação anterior 17.

\section{Variável dependente}

Os indivíduos foram classificados como hipertensos quando apresentaram médias de PA sistólica/ diastólica iguais ou superiores a 140/90mmHg 1, e/ou relataram diagnóstico médico prévio da doença, além do uso de medicamento anti-hipertensivo. Tendo em vista o seguimento dos participantes do estudo ao longo do tempo, em três momentos distintos de coleta de dados a variável dependente foi definida com base na presença de hipertensão ( $\operatorname{sim} /$ não) em, pelo menos, uma das ocasiões. A análise específica de casos incidentes de hipertensão é objeto de outra investigação em curso e será publicada oportunamente.

\section{Exposições}

Os anos de educação foram utilizados como indicador de posição socioeconômica tanto dos participantes (PSE atual ou PSEa) como de seus pais (PSE na infância ou PSEi). A educação é um indicador de posição socioeconômica amplamente usado em estudos epidemiológicos, pois geralmente está disponível para ambos os sexos, é uma medida quantificável e, especialmente, mais estável ao longo do tempo, se comparada com a ocupação e a renda 18 .

A MEI foi calculada pela variação da PSEa dos indivíduos em relação à PSEi de seus pais. Foram definidas três variáveis de MEI, uma referente à educação paterna, outra, à educação materna e, finalmente, à educação familiar. A MEI familiar foi criada com base na maior mobilidade entre a MEI paterna e a materna. Assim, se a mobilidade paterna foi baixa e a materna descendente, a variável de MEI familiar resultante foi constituída da mobilidade materna. Se a mobilidade paterna foi ascendente e a materna descendente, a variável de MEI familiar refletiu a mobilidade paterna e assim por diante. 
As categorias da variável de MEI foram assim constituídas: baixa (referindo-se aos participantes de baixa escolaridade, cujos pais também apresentavam nível educacional baixo); alta (em relação aos indivíduos e pais com alta escolarização); ascendente (para indicar entrevistados cuja escolaridade é alta, em oposição a seus pais); e descendente (com o intuito de refletir a situação de membros do estudo que tiveram menos escolarização do que seus próprios pais). A categorização das variáveis de posição socioeconômica foi realizada com base na divisão dos níveis de educação no Brasil: Ensino Fundamental (0-9 anos), Ensino Médio (10-12 anos) e Ensino Superior (> 12 anos) 19. Essa divisão entrou em vigor no país apenas em 1996. Anteriormente, a educação média finalizava ao serem completados 11 anos de estudos. Visando a reduzir erros de classificação, indivíduos com menos de 30 anos, ou seja, com 16 anos ou menos em 1996, foram classificados segundo a nova divisão. Indivíduos com 30 anos ou mais, ou seja, com 17 anos ou mais em 1996, foram classificados segundo a divisão anterior.

Alguns autores indicam que os processos sociais envolvidos na transição entre a educação fundamental e a média são menos marcantes do que entre a educação média e a superior, momento em que os indivíduos recebem maior carga de responsabilidades, muitas vezes iniciando a vida longe dos pais e enfrentando novas situações e riscos à saúde 20. Os níveis de educação fundamental e médio foram, dessa forma, condensados em uma única categoria de posição socioeconômica, a baixa. A educação superior constituiu a categoria alta escolaridade da posição socioeconômica.

No que se refere à PSEi, levou-se em consideração a melhora da educação no Brasil ao longo das últimas décadas 21. Assim, a PSEi foi categorizada de acordo com a mediana dos anos de educação dos pais em relação à idade dos participantes do estudo. Aqueles pais com nível educacional abaixo da mediana segundo a idade dos participantes foram classificados como educação baixa. Os pais com nível de educação acima da mediana foram classificados como alta educação.

\section{Variáveis de confusão e interação}

As variáveis de confusão sexo, idade (20-29, 30-39, 40-49, 50+ anos) e renda total do domicílio (quintis) foram testadas no procedimento de definição dos modelos finais, considerando-se que a literatura indica frequente associação destas com as variáveis de interesse 2,22. As análises foram ajustadas também para o efeito do tempo decorrido entre as ondas. A discriminação interpessoal foi mensurada por meio da Escala de Discriminação Explícita (EDE), a qual permite analisar tratamentos injustos ao longo da vida ocorridos em diferentes domínios, bem como as motivações para a discriminação e a classificação daquele evento como discriminatório ou não pelo participante do estudo. A EDE foi elaborada para avaliar os efeitos da discriminação sobre desfechos de saúde e comportamentos 23 . A cor da pele/"raça" foi observada pelo entrevistador (heteroclassificação) porque, segundo sugerem alguns autores 24 , reflete como os participantes são “percebidos” por terceiros. Esse processo também pode ser chamado de fisiognomia e se refere a um julgamento com base na aparência, que pode determinar o grau de exposição a riscos de saúde 24 .

\section{Análises estatísticas}

As análises multinível foram executadas no software Stata 14 (https://www.stata.com), utilizando-se o programa GLLAMM - generalized linear latent and mixed models para a obtenção do intercepto e do coeficiente aleatórios com desfecho dicotômico (apresentar ou não HAS). Os modelos multinível finais foram definidos por estratégia forward ( $\mathrm{p}<0,05)$, inserindo cada uma das variáveis de confusão (sexo, idade, renda e tempo decorrido entre as ondas do estudo) e interação (discriminação interpessoal e cor da pele/"raça"), comparando sequencialmente os modelos aninhados por teste de máxima verossimilhança. A análise dos modelos GLLAMM com e sem gradiente aleatório também foi realizada. A interação entre discriminação e MEI foi testada em análise estratificada por cor da pele/"raça", criando-se uma nova variável resultante da multiplicação entre discriminação interpessoal (dicotômica) e MEI (politômica). Nesses modelos de interação, considerou-se o papel da discriminação sobre as desigualdades raciais em educação 13 e sua ligação com a PA de adultos 6,7. A hipótese testada levou em conta que o efeito da MEI poderia ser intensificado em situações de discriminação interpessoal, especialmente em negros e pardos. Todas as análises estatísticas levaram em consideração a estrutura e os pesos amostrais do estudo. 


\section{Resultados}

A amostra foi composta por maioria feminina, abaixo de 40 anos, branca, no $4^{\circ}$ quintil de renda total do domicílio, com PSEa e PSEi baixas. As perdas ocorreram, principalmente, em função de os participantes terem se mudado para outros municípios, não terem sido localizados em seus domicílios ou terem recursado permanecer na pesquisa. A Tabela 1 apresenta a distribuição dos participantes conforme características sociodemográficas na linha de base (2009), incluindo o relato de experiências discriminatórias ao longo da vida (2012) e a prevalência de HAS. Observou-se maior prevalência da doença entre homens, respondentes acima de 50 anos, classificados como negros ou pardos, do $3 \underline{0}$ quintil de renda, com PSEi e PSEa baixas e com MEI descendente e baixa. A prevalência de HAS foi similar entre os que relataram discriminação e os que não relataram.

Tabela 1

Descrição da amostra de acordo com as características sociodemográficas e com a prevalência de hipertensão.

Florianópolis, Santa Catarina, Brasil, 2009.

\begin{tabular}{|c|c|c|c|c|}
\hline \multirow[t]{2}{*}{ Variáveis } & \multirow{2}{*}{$\begin{array}{c}\text { Total }(n=1.720) \\
\%\end{array}$} & \multicolumn{3}{|c|}{ Hipertensão } \\
\hline & & $\%$ & IC95\% & Valor de $p$ * \\
\hline \multicolumn{5}{|l|}{ Sexo } \\
\hline Feminino & 56,03 & 36,48 & 33,$41 ; 39,65$ & $<0,001$ \\
\hline Masculino & 43,97 & 55,36 & 51,$76 ; 58,90$ & \\
\hline \multicolumn{5}{|l|}{ Idade (anos) } \\
\hline $20-29$ & 30,36 & 32,89 & 28,$94 ; 37,10$ & $<0,001$ \\
\hline 30-39 & 22,43 & 38,35 & 33,$53 ; 43,42$ & \\
\hline $40-49$ & 26,39 & 50,43 & 45,$72 ; 55,13$ & \\
\hline $50+$ & 20,82 & 61,69 & 56,$41 ; 66,72$ & \\
\hline \multicolumn{5}{|l|}{ Cor da pele/“Raça” } \\
\hline Negros e pardos & 10,51 & 51,35 & 44,$04 ; 58,60$ & 0,067 \\
\hline Brancos & 89,49 & 44,11 & 41,$60 ; 46,66$ & \\
\hline \multicolumn{5}{|l|}{ Discriminação $(n=1.183)$ ** } \\
\hline Não & 46,11 & 46,95 & 42,$73 ; 51,22$ & 0,941 \\
\hline $\operatorname{Sim}$ & 53,89 & 46,97 & 43,$05 ; 50,93$ & \\
\hline \multicolumn{5}{|l|}{ Renda total do domicílio } \\
\hline 1ㅇq quintil & 19,88 & 45,24 & 39,$92 ; 50,66$ & 0,190 \\
\hline 2o quintil & 19,92 & 47,71 & 42,$28 ; 53,19$ & \\
\hline 3o quintil & 20,66 & 47,93 & 42,$56 ; 53,35$ & \\
\hline 4o quintil & 22,35 & 43,27 & 38,$24 ; 48,43$ & \\
\hline 5o quintil & 17,16 & 39,04 & 33,$53 ; 44,85$ & \\
\hline \multicolumn{5}{|l|}{ Educação na fase adulta (PSEa) } \\
\hline Baixa & 58,83 & 50,08 & 46,$95 ; 53,22$ & $<0,001$ \\
\hline Alta & 41,17 & 37,38 & 33,$81 ; 41,10$ & \\
\hline \multicolumn{5}{|l|}{ Educação na infância (PSEi) ** } \\
\hline \multicolumn{5}{|l|}{ Educação paterna } \\
\hline Baixa ( $\leq$ mediana de anos de estudos) & 55,07 & 45,24 & 41,$11 ; 49,44$ & 0,778 \\
\hline Alta (> mediana de anos de estudos) & 44,93 & 44,17 & 39,$58 ; 48,87$ & \\
\hline \multicolumn{5}{|l|}{ Educação materna } \\
\hline Baixa ( $\leq$ mediana de anos de estudos) & 54,91 & 46,02 & 41,$97 ; 50,13$ & 0,833 \\
\hline Alta (> mediana de anos de estudos) & 45,09 & 45,94 & 41,$40 ; 50,55$ & \\
\hline
\end{tabular}

(continua) 
Tabela 1 (continuação)

\begin{tabular}{|c|c|c|c|c|}
\hline \multirow[t]{2}{*}{ Variáveis } & \multirow{2}{*}{$\begin{array}{c}\text { Total }(n=1.720) \\
\%\end{array}$} & \multicolumn{3}{|c|}{ Hipertensão } \\
\hline & & $\%$ & IC95\% & Valor de $p$ * \\
\hline \multicolumn{5}{|l|}{ Educação na infância (PSEi) ** } \\
\hline \multicolumn{5}{|l|}{ Educação familiar *** } \\
\hline Baixa ( $\leq$ mediana de anos de estudos) & 48,61 & 47,48 & 43,$27 ; 51,73$ & 0,443 \\
\hline Alta (> mediana de anos de estudos) & 51,39 & 45,40 & 41,$28 ; 49,58$ & \\
\hline \multicolumn{5}{|l|}{ MEI } \\
\hline \multicolumn{5}{|l|}{ Paterna } \\
\hline Baixa (baixa $\rightarrow$ baixa) & 36,31 & 47,31 & 42,$19 ; 52,50$ & $<0,001$ \\
\hline Descendente (alta $\rightarrow$ baixa) & 15,30 & 57,28 & 49,$16 ; 65,03$ & \\
\hline Ascendente (baixa $\rightarrow$ alta) & 18,83 & 41,53 & 34,$63 ; 48,80$ & \\
\hline Alta (alta $\rightarrow$ alta) & 29,56 & 37,21 & 31,$78 ; 42,98$ & \\
\hline \multicolumn{5}{|l|}{ Materna } \\
\hline Baixa (baixa $\rightarrow$ baixa) & 37,19 & 47,82 & 42,$87 ; 52,80$ & $<0,001$ \\
\hline Descendente (alta $\rightarrow$ baixa) & 15,50 & 60,56 & 52,$55 ; 68,03$ & \\
\hline Ascendente (baixa $\rightarrow$ alta) & 17,79 & 42,56 & 35,$53 ; 49,90$ & \\
\hline Alta (alta $\rightarrow$ alta) & 29,53 & 38,24 & 32,$88 ; 43,90$ & \\
\hline \multicolumn{5}{|l|}{ Familiar } \\
\hline Baixa (baixa $\rightarrow$ baixa) & 35,77 & 49,21 & 44,$28 ; 54,15$ & $<0,001$ \\
\hline Descendente (alta $\rightarrow$ baixa) & 18,93 & 58,09 & 51,$11 ; 64,75$ & \\
\hline Ascendente (baixa $\rightarrow$ alta) & 12,88 & 43,04 & 34,$99 ; 51,47$ & \\
\hline Alta (alta $\rightarrow$ alta) & 32,43 & 37,94 & 32,$97 ; 43,17$ & \\
\hline
\end{tabular}

IC95\%: intervalo de 95\% de confiança; MEl: mobilidade educacional intergeracional; PSEa: posição socieconômica na fase adulta; PSEi: posição socieconômica na infância.

* Teste de qui-quadrado de Pearson para a comparação das prevalências de hipertensão entre as categorias das variáveis sociodemográficas;

** Ano de 2012;

*** Mobilidade mais favorável entre os pais.

As comparações entre os modelos com e sem coeficiente aleatório não demonstraram acréscimo de informação com a inclusão do gradiente, então, optou-se pelos modelos apenas com o intercepto aleatório. Foram selecionados três modelos finais, testando hierarquicamente o efeito da MEI ao longo da vida sobre a HAS. A análise multinível com efeitos aleatórios mostrou que a influência da MEI foi mantida mesmo após o ajuste para os fatores de confusão, como mostrado na Tabela 2. A posição socioeconômica constantemente baixa entre as gerações (MEI baixa) foi considerada a categoria de referência. Observou-se que a MEI descendente aumentou a odds de HAS entre os participantes. Esse comportamento foi observado tanto no modelo 1 como no 2 e no modelo 3. No sentido oposto, a MEI ascendente reduziu a odds de HAS tanto quando foi testada a mobilidade paterna, modelo 1, materna, modelo 2 e familiar, modelo 3. A permanência na PSE elevada entre gerações (MEI alta) foi capaz de reduzir de modo estatisticamente significativo a odds de HAS em todos os modelos. Ainda na Tabela 2, considerando-se a influência dos efeitos aleatórios, não mensurados pelas variáveis do estudo, as correlações intraclasse (CIC) para os interceptos aleatórios nos três modelos $(0,91)$ indicaram elevada propensão de os participantes se manterem em seu estado inicial ao longo do estudo, devido às características individuais. Tanto a idade como o sexo e o tempo de acompanhamento apresentaram elevada influência sobre a odds de HAS.

$\mathrm{Na}$ análise da interação entre discriminação e MEI, o grupo que não relatou experiências de discriminação foi considerado a categoria de referência. Conforme descrito na Tabela 3, a relação inversa entre MEI e presença de HAS foi mantida entre os indivíduos que relataram discriminação, quando comparados aos que não relataram. Em praticamente todos os modelos de interação testados, a odds de HAS aumentou em MEI baixa e descendente entre os indivíduos que relataram discriminação em relação aos que não relataram, exceto na categoria descendente do modelo paterno. Nos três modelos, 


\section{Tabela 2}

Modelos mistos e generalizados (generalized linear latent and mixed models) para o exame da associação entre as variáveis independentes de interesse e hipertensão. Florianópolis, Santa Catarina, Brasil, 2009, 2012 e 2014.

\begin{tabular}{|c|c|c|c|c|c|c|c|c|c|}
\hline & \multicolumn{9}{|c|}{ Hipertensão } \\
\hline & \multicolumn{3}{|c|}{ Modelo 1 (Pai) } & \multicolumn{3}{|c|}{ Modelo 2 (Mãe) } & \multicolumn{3}{|c|}{ Modelo 3 (Familiar) } \\
\hline & OR & IC95\% & Valor de p & OR & IC95\% & Valor de p & OR & IC95\% & Valor de $p$ \\
\hline \multicolumn{10}{|l|}{ Efeitos fixos } \\
\hline \multicolumn{10}{|l|}{ Mobilidade de posição } \\
\hline \multicolumn{10}{|l|}{ socioeconômica } \\
\hline Baixa (baixa $\rightarrow$ baixa) & 1,00 & - & - & 1,00 & - & - & 1,00 & - & - \\
\hline Descendente (alta $\rightarrow$ baixa) & 1,13 & 0,$44 ; 2,91$ & 0,797 & 1,50 & 0,$57 ; 3,95$ & 0,412 & 1,14 & $049 ; 2,64$ & 0,757 \\
\hline Ascendente (baixa $\rightarrow$ alta) & 0,72 & 0,$32 ; 1,62$ & 0,432 & 0,82 & 0,$37 ; 1,80$ & 0,623 & 0,86 & 0,$36 ; 2,02$ & 0,724 \\
\hline Alta (alta $\rightarrow$ alta) & 0,39 & 0,$20 ; 0,76$ & 0,006 & 0,35 & 0,$18 ; 0,69$ & 0,002 & 0,35 & 0,$19 ; 0,66$ & 0,001 \\
\hline \multicolumn{10}{|l|}{ Discriminação } \\
\hline Não & 1,00 & - & - & 1,00 & - & - & 1,00 & - & - \\
\hline Sim & 1,22 & 0,$67 ; 2,20$ & 0,519 & 1,28 & 0,$72 ; 2,26$ & 0,395 & 1,26 & 0,$71 ; 2,24$ & 0,430 \\
\hline \multicolumn{10}{|l|}{ Cor da pele/“Raça” } \\
\hline Negros e pardos & 1,00 & - & - & 1,00 & - & - & 1,00 & - & - \\
\hline Brancos & 0,47 & 0,$17 ; 1,26$ & 0,134 & 0,44 & 0,$18 ; 1,12$ & 0,085 & 0,45 & 0,$18 ; 1,16$ & 0,098 \\
\hline \multicolumn{10}{|l|}{ Sexo } \\
\hline Feminino & 1,00 & - & - & 1,00 & - & - & 1,00 & - & - \\
\hline Masculino & 81,73 & 25,$60 ; 260,95$ & $<0,001$ & 88,41 & 28,$35 ; 275,69$ & $<0,001$ & 87,04 & 28,$29 ; 267,75$ & $<0,001$ \\
\hline \multicolumn{10}{|l|}{ Idade (anos) } \\
\hline $20-29$ & 1,00 & - & - & 1,00 & - & - & 1,00 & - & - \\
\hline $30-39$ & 23,93 & 4,$98 ; 114,92$ & $<0,001$ & 26,97 & 6,$06 ; 120,10$ & $<0,001$ & 27,74 & 6,$32 ; 121,75$ & $<0,001$ \\
\hline $40-49$ & 32,22 & 7,$65 ; 135,59$ & $<0,001$ & 35,52 & 9,$08 ; 138,92$ & $<0,001$ & 36,57 & 9,$61 ; 139,22$ & $<0,001$ \\
\hline $50+$ & 110,49 & 23,$92 ; 510,38$ & $<0,001$ & 122,02 & 28,$71 ; 518,45$ & $<0,001$ & 122,60 & 29,$58 ; 508,14$ & $<0,001$ \\
\hline Tempo & 1,18 & 1,$13 ; 1,24$ & $<0,001$ & 1,18 & 1,$13 ; 1,24$ & $<0,001$ & 1,18 & 1,$13 ; 1,24$ & $<0,001$ \\
\hline Intercepto * & 0,01 & 0,$00 ; 0,46$ & $<0,001$ & 0,01 & 0,$00 ; 0,04$ & $<0,001$ & 0,01 & 0,$00 ; 0,042$ & $<0,001$ \\
\hline \multicolumn{10}{|l|}{ Efeitos randômicos ** } \\
\hline \multicolumn{10}{|l|}{ Intercepto } \\
\hline Variância & & 32,51 & & & 33,31 & & & 33,37 & \\
\hline $\mathrm{CIC}$ & & 0,91 & & & 0,91 & & & 0,91 & \\
\hline Log-likelihood & & $-239,12$ & & & $-239,18$ & & & $-239,05$ & \\
\hline
\end{tabular}

IC95\%: intervalo de 95\% de confiança; CIC: correlação intraclasse; OR: odds ratio.

* Intercepto da regressão;

** Variância no nível individual.

indivíduos relatando discriminação apresentaram odds de HAS mais baixa em MEI ascendente e alta, sendo que os resultados na categoria alta foram estatisticamente significativos.

Entretanto, analisando graficamente a interação entre MEI e discriminação, essa tendência inversa citada antes praticamente desapareceu. Observou-se redução da odds de HAS em quase todas as categorias de MEI quando a discriminação foi relatada, e isto foi repetido em todos os três modelos testados (Figuras 1a, 1b e 1c). As únicas exceções foram observadas na categoria descendente dos modelos materno (Figura 1a) e familiar (Figura 1c). 
Modelos mistos e generalizados (generalized linear latent and mixed models) para o exame da associação entre as variáveis independentes de interesse e suas interações com hipertensão. Florianópolis, Santa Catarina, Brasil, 2009, 2012 e 2014.

\begin{tabular}{|c|c|c|c|c|c|c|c|c|c|}
\hline & \multicolumn{9}{|c|}{ Hipertensão } \\
\hline & \multicolumn{3}{|c|}{ Modelo 4 (Pai) } & \multicolumn{3}{|c|}{ Modelo 5 (Mãe) } & \multicolumn{3}{|c|}{ Modelo 6 (Familiar) } \\
\hline & OR & IC95\% & Valor de $p$ & OR & IC95\% & Valor de $p$ & OR & IC95\% & Valor de $p$ \\
\hline \multicolumn{10}{|l|}{ Efeitos fixos } \\
\hline \multicolumn{10}{|l|}{ MEI X Discriminação } \\
\hline Não discriminados & 1,00 & - & - & 1,00 & - & - & 1,00 & - & - \\
\hline \multicolumn{10}{|l|}{ Discriminados } \\
\hline Baixa (baixa $\rightarrow$ baixa) & 1,42 & 0,$62 ; 3,23$ & 0,403 & 1,31 & 0,$57 ; 3,02$ & 0,526 & 1,29 & 0,$57 ; 2,91$ & 0,533 \\
\hline Descendente (alta $\rightarrow$ baixa) & 0,89 & 0,$27 ; 2,96$ & 0,851 & 1,90 & 0,$53 ; 6,83$ & 0,325 & 1,60 & 0,$50 ; 5,08$ & 0,425 \\
\hline Ascendente (baixa $\rightarrow$ alta) & 0,45 & 0,$18 ; 1,11$ & 0,082 & 0,64 & 0,$28 ; 1,47$ & 0,295 & 0,65 & 0,$26 ; 1,66$ & 0,371 \\
\hline Alta (alta $\rightarrow$ alta) & 0,40 & 0,$18 ; 0,87$ & 0,022 & 0,34 & 0,$16 ; 0,74$ & 0,007 & 0,36 & 0,$18 ; 0,73$ & 0,005 \\
\hline \multicolumn{10}{|l|}{ Cor da pele/"Raça" } \\
\hline Negros e pardos & 1,00 & - & - & 1,00 & - & - & 1,00 & - & - \\
\hline Brancos & 0,55 & 0,$15 ; 2,05$ & 0,376 & 0,51 & 0,$18 ; 1,39$ & 0,187 & 0,50 & 0,$18 ; 1,41$ & 0,191 \\
\hline \multicolumn{10}{|l|}{ Sexo } \\
\hline Feminino & 1,00 & - & - & 1,00 & - & - & 1,00 & - & - \\
\hline Masculino & 98,69 & 28,$29 ; 344,34$ & $<0,001$ & 103,97 & 29,$06 ; 371,94$ & $<0,001$ & 101,38 & 28,$76 ; 357,38$ & $<0,001$ \\
\hline \multicolumn{10}{|l|}{ Idade (anos) } \\
\hline $20-29$ & 1,00 & - & - & 1,00 & - & - & 1,00 & - & - \\
\hline $30-39$ & 26,60 & 5,$13 ; 137,95$ & $<0,001$ & 27,55 & 5,$61 ; 135,24$ & $<0,001$ & 27,38 & 5,$56 ; 134,73$ & $<0,001$ \\
\hline $40-49$ & 39,39 & 9,$61 ; 161,42$ & $<0,001$ & 40,43 & 9,$66 ; 169,19$ & $<0,001$ & 39,86 & 9,$68 ; 164,15$ & $<0,001$ \\
\hline $50+$ & 141,17 & 34,$01 ; 586,03$ & $<0,001$ & 139,97 & 33,$41 ; 579,81$ & $<0,001$ & 138,17 & 33,$37 ; 572,05$ & $<0,001$ \\
\hline Tempo & 1,19 & 1,$13 ; 1,25$ & $<0,001$ & 1,19 & 1,$13 ; 1,25$ & $<0,001$ & 1,19 & 1,$13 ; 1,25$ & $<0,001$ \\
\hline Intercepto * & 0,00 & 0,$00 ; 0,03$ & $<0,001$ & 0,00 & 0,$00 ; 0,33$ & $<0,001$ & 0,00 & 0,$00 ; 0,03$ & $<0,001$ \\
\hline \multicolumn{10}{|l|}{ Efeitos randômicos ** } \\
\hline \multicolumn{10}{|l|}{ Intercepto } \\
\hline Variância & & 33,23 & & & 33,34 & & & 33,20 & \\
\hline $\mathrm{CIC}$ & & 0,91 & & & 0,91 & & & 0,91 & \\
\hline Log-likelihood & & $-240,63$ & & & $-240,51$ & & & $-240,53$ & \\
\hline
\end{tabular}

IC95\%: intervalo de 95\% de confiança; CIC: correlação intraclasse; MEl: mobilidade educacional intergeracional; OR: odds ratio.

* Intercepto da regressão;

** Variância no nível individual.

Estratificando-se as análises por cor da pele/“raça”, não foi observada modificação de efeito no modelo materno entre negros ou pardos e brancos (Figura 2). Mas, nos modelos paterno (Figura 3) e familiar (Figura 4), observou-se diferença na odds de HAS entre negros ou pardos e brancos em MEI baixa e descendente. Enquanto no modelo paterno a odds de HAS aumentou entre indivíduos negros ou pardos que relataram discriminação em MEI baixa e descendente, a OR reduziu em indivíduos brancos na mesma situação. Já no modelo familiar, a odds de HAS não variou na categoria baixa e aumentou na categoria descendente entre negros ou pardos que relataram discriminação em relação aos que não relataram, mas diminuiu entre brancos na mesma situação nas categorias baixa e descendente. Em MEI ascendente e alta, a odds de HAS foi menor entre aqueles que relataram discriminação em todos os modelos e entre negros ou pardos e brancos. 
Figura 1

Análises da interação entre discriminação e mobilidade educacional intergeracional (MEI).

1a) Modelo materno

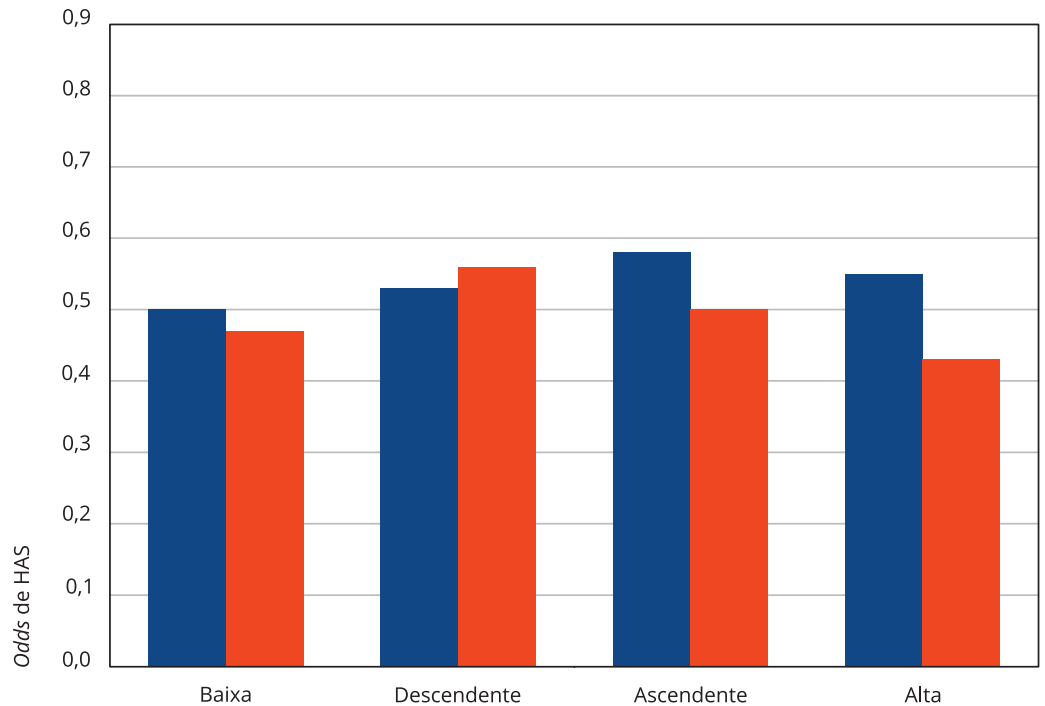

Não discriminados

Discriminados

1b) Modelo paterno

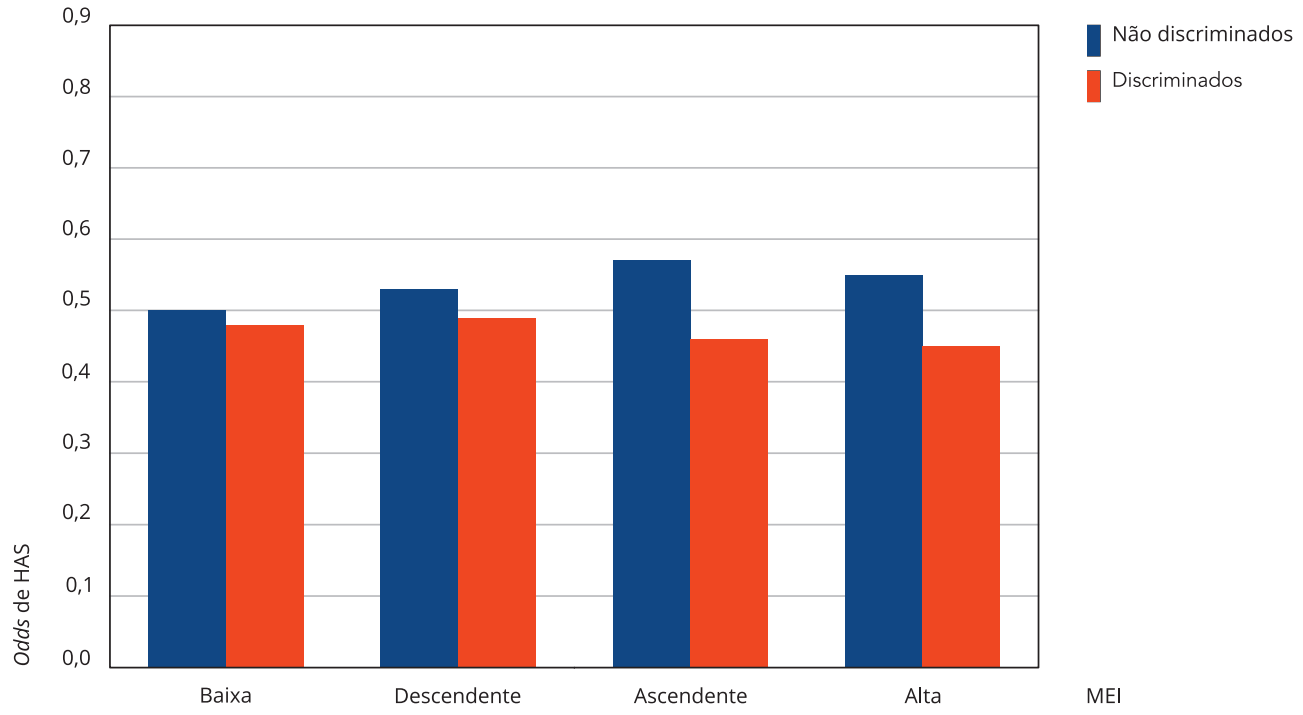

(continua) 
Figura 1 (continuação)

1c) Modelo familiar

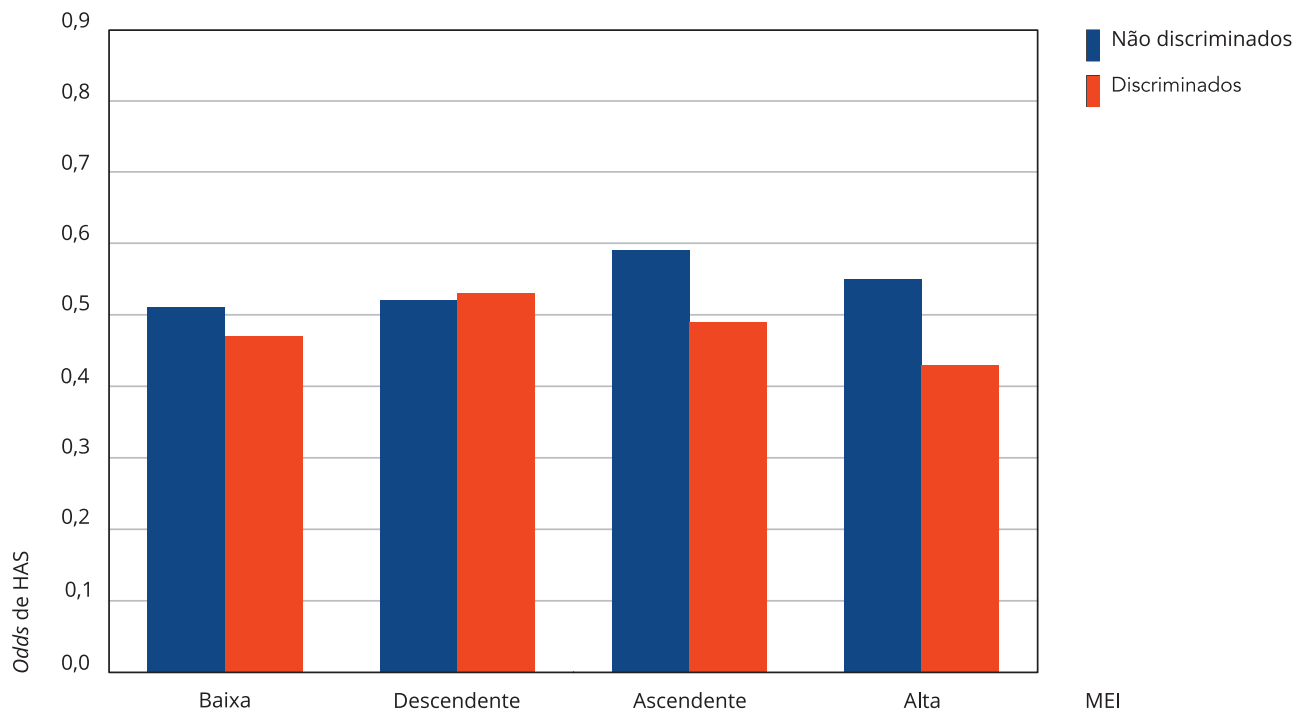

HAS: hipertensão arterial sistólica.

Figura 2

Análises da interação entre discriminação, mobilidade educacional intergeracional (MEl) e cor da pele/"raça", modelo materno.

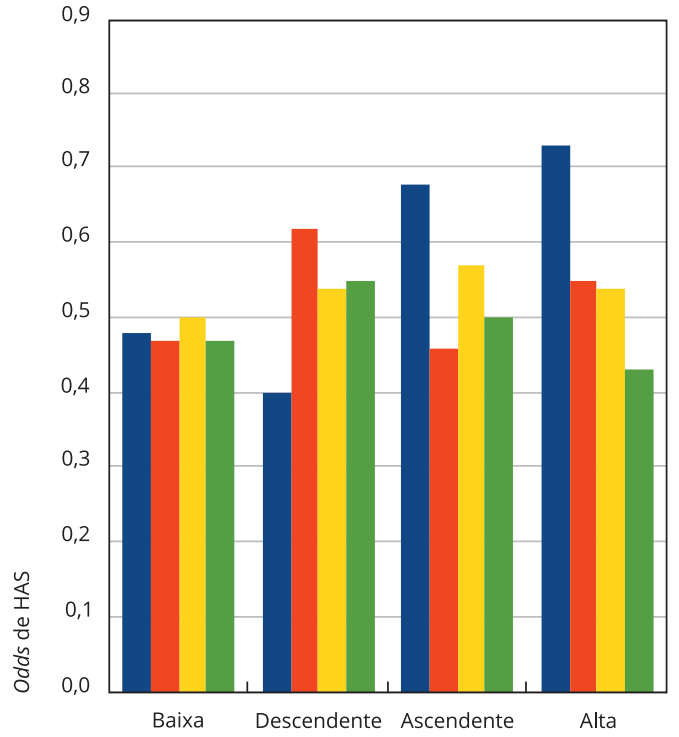

Negros ou pardos não discriminados

Negros ou pardos discriminados

$\square$ Brancos não discriminados

Brancos discriminados 
Figura 3

Análises da interação entre discriminação, mobilidade educacional intergeracional (MEI) paterna e cor da pele/"raça", modelo paterno.

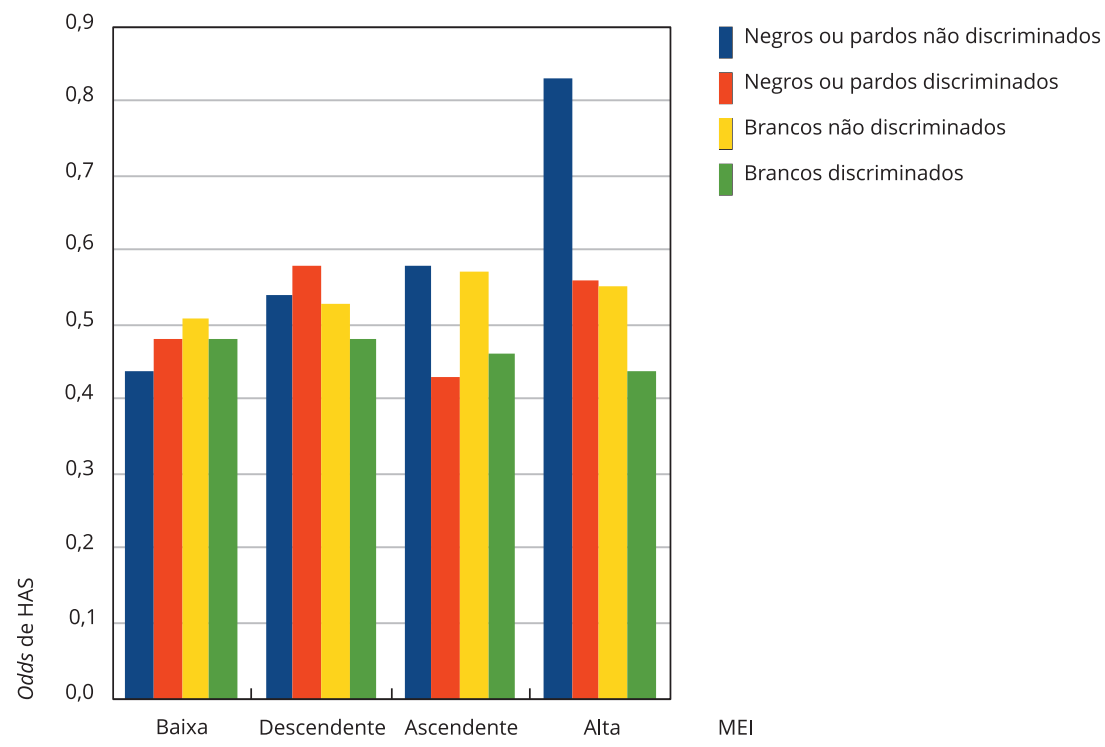

HAS: hipertensão arterial sistólica.

\section{Figura 4}

Análises da interação entre discriminação, mobilidade educacional intergeracional (MEI) familiar e cor da pele/"raça", modelo familiar.

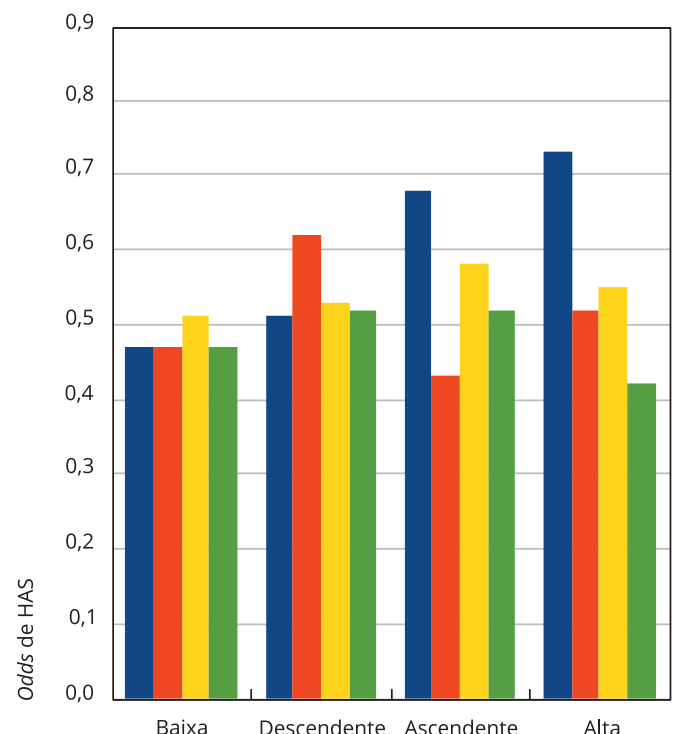

Negros ou pardos não discriminados

Negros ou pardos discriminados

$\|$ Brancos não discriminados

Brancos discriminados

MEI

HAS: hipertensão arterial sistólica. 


\section{Discussão}

O presente artigo analisou a influência da MEI sobre a ocorrência de HAS em adultos de uma cidade do Sul do Brasil. Os resultados da análise longitudinal multinível com efeitos aleatórios demonstraram que a permanência na posição socioeconômica elevada (MEI alta) foi capaz de reduzir de modo estatisticamente significativo a odds de HAS em todos os modelos. A interação entre discriminação interpessoal e MEI também foi testada em uma análise estratificada por cor da pele/"raça”, a qual evidenciou que a odds de HAS aumenta na categoria descendente de MEI entre negros ou pardos que relataram discriminação em relação aos que não relataram.

A elevada prevalência de HAS observada $(44,9 \%)$ se aproxima da prevalência observada na América Latina e Caribe por outros autores (39,1\%, IC95\%: 33,1; 45,2) 25. Entretanto, excede as demais prevalências encontradas em outros estudos realizados no Brasil, tais como a Pesquisa Nacional de Saúde de 2013 (22,9\% na Região Sul) 26 e a pesquisa Vigitel Brasil 2017 Saúde Suplementar (21,5\% em Florianópolis) 27. A diferença é que os dados dos dois últimos estudos citados se referem apenas aos indivíduos que relataram diagnóstico médico prévio de HAS, e, no presente estudo, considerou-se o uso de medicamentos anti-hipertensivos (prevalência de $6 \%$ na linha de base) e a média de duas aferições de PA sistólica/diastólica, o que pode ter contribuído para a maior prevalência observada.

Entre os indivíduos participantes do presente trabalho, a hipertensão entre negros e pardos $(51,3 \%)$ foi mais frequente do que entre brancos $(44,1 \%)$, corroborando com os resultados encontrados por outros autores 6 . Vale ressaltar que em países racialmente desiguais como o Brasil, a cor/"raça" representa um indicador de classe social e racismo estrutural, além de atuar como motivador para discriminação interpessoal, afetando a saúde de forma desfavorável 28. Um estudo realizado em Cuba, país caracterizado por menor desigualdade racial, não foi encontrada diferença na prevalência de HAS entre negros e brancos 29 .

Não foram observadas diferenças relevantes na prevalência de HAS entre os quintis de renda total do domicílio; entre os níveis educacionais na infância e entre indivíduos que vivenciaram ou não experiências discriminatórias. É importante destacar que, embora as prevalências de HAS tenham sido semelhantes nesses grupos, pesquisas têm sido realizadas no intuito de demonstrar que a discriminação experimentada ao longo da vida 6,28, bem como privações materiais desde a infância 30, assim como a baixa escolaridade dos pais 31 podem influenciar a PA e a saúde na vida adulta. Na amostra analisada, a HAS foi mais prevalente entre indivíduos com escolaridade baixa, reforçando o que foi observado em estudos prévios 2,5,6. Em adição, indivíduos com MEI descendente também apresentaram maior prevalência da doença, seguidos pelos que mantiveram o nível educacional baixo ao longo da vida. Um estudo longitudinal realizado na Suécia com pares de gêmeos 32 e outro transversal realizado nos Estados Unidos com homens negros 33 observaram que a prevalência de HAS foi mais elevada entre indivíduos que permaneceram em PSE baixa ao longo da vida.

Os efeitos fixos da análise longitudinal multinível demonstraram que apenas a MEI elevada foi capaz de reduzir de forma estatisticamente significativa a odds de HAS em relação àqueles indivíduos com MEI baixa. Esse dado torna-se preocupante quando se considera a tendência existente no Brasil de persistência educacional em extremos sociais, o que dificulta a mudança de PSE dos participantes em relação aos seus pais quando a PSEi é baixa 12,13. Além disso, mesmo havendo ascensão educacional intergeracional, ela não surtiu o mesmo efeito favorável de manter-se em PSE elevada ao longo da vida. Corroborando com os resultados encontrados no presente estudo, em que pese as diferenças metodológicas e contextuais, uma pesquisa estadunidense transversal realizada com adultos afrodescendentes encontrou associação entre PSE alta constante ao longo da vida e HAS (OR = 7,27; IC95\%: $1,91 ; 27,51)$ em relação à PSE constantemente baixa 33. Alguns autores sugerem que a escolaridade pode afetar a saúde por meio de um sistema de desvantagens cumulativas, referentes a comportamentos, com impacto sobre a saúde, sobre a prevenção e o tratamento de doenças, bem como por meio de ambientes de trabalho estressantes e do contexto socioeconômico da vizinhança residencial 34,35,36.

Adicionalmente, de acordo com os efeitos aleatórios, a CIC do intercepto aleatório $(0,91$ nos três modelos) mostrou que uma grande parte da variância do desfecho, tanto no modelo paterno (modelo 1), como nos materno (modelo 2) e familiar (modelo 3), pode ser explicada por características individuais dos participantes, as quais são imutáveis ao longo do estudo e não mensuradas no modelo testado. Alguns autores sugerem, por exemplo, que o aleitamento materno e o peso ao nascer podem trazer 
efeitos latentes que têm o poder de influenciar a PA na idade adulta 22,31. Além disso, situações de estresse psicossocial, tais como privação material na infância, na adolescência e juventude também podem atuar sobre a PA na vida adulta 30,37 .

As análises da interação entre discriminação interpessoal e MEI mostraram redução da odds de HAS em praticamente todas as categorias de MEI em situação de discriminação relatada e em todos os modelos. Esse efeito pode ser resultado de opressão internalizada 38, perspectiva que afirma que os indivíduos nem sempre relatam aquilo que vivenciam, apesar das manifestações somáticas (embodiment) expressadas pelo corpo demonstrarem o contrário. Assim, indivíduos que não relataram discriminação por não se perceberem vítimas desses atos, ainda que tenham sido, podem ter constituído a categoria de referência "não discriminados", levando a esse resultado contraditório.

A inconsistência dos resultados encontrados na literatura, relacionando a discriminação interpessoal à HAS, também pode ser devida ao não relato e ao não reconhecimento de determinados tratamentos como discriminatórios. As evidências mais consistentes encontradas relacionam a discriminação à PA ambulatorial, a qual pode ser considerada um indicador de reação imediata ao estresse e não dependente do entendimento e do relato dos indivíduos com relação ao ato discriminatório 28. Vale ressaltar ainda que a redução da odds de HAS entre os indivíduos que relataram discriminação não significa um efeito benéfico da discriminação sobre a PA. Tampouco legitima a injustiça social e a aceitação das desigualdades sociais em HAS e em saúde, de maneira mais ampla. Mas evidencia a necessidade de compreender como a discriminação sofrida é percebida, relatada e como impacta a saúde dos indivíduos. Além disso, a busca por soluções para conter a discriminação independe do motivo que a desencadeia e se sobrepõe aos resultados de estudos científicos.

Observaram-se, igualmente, diferenças no comportamento da odds de HAS nos modelos paterno e materno, entre negros ou pardos, em MEI baixa, e entre brancos, no modelo materno, em MEI descendente, indicando que a mobilidade medida com base no pai pode ter um efeito diferente da mobilidade medida baseando-se na mãe. No Brasil, país caracterizado pelo relevante número de famílias monoparentais chefiadas por mulheres $(16,3 \%) 39$, sugere-se relevante influência materna sobre comportamentos em saúde e crenças na vida adulta, embora sejam necessários mais estudos analisando o problema sob esta perspectiva.

\section{Conclusão}

Os resultados encontrados no presente artigo reforçam a importância da educação para HAS em adultos do Sul do Brasil em uma perspectiva de curso de vida. Observou-se que, entre gerações, manter a escolaridade elevada dos pais favoreceu a redução da HAS, ainda que variáveis aleatórias também influenciem o aumento da PA. A MEI ascendente não reverteu de forma significativa os efeitos da posição socioeconômica desfavorável na infância. Nesse sentido, reitera-se a necessidade de políticas públicas de longo prazo, visando ao acesso continuado à educação no país e também à melhoria da saúde da população.

Os resultados da análise estratificada por cor/"raça" sugerem que situações de discriminação podem atuar conjuntamente com a MEI desfavorável, elevando a odds de HAS, especialmente entre negros e pardos. Esse resultado oferece ainda maior subsídio ao argumento de que a hipertensão e suas desigualdades sociais devem ser enfrentadas, entre outras, com políticas públicas que privilegiem a educação: esta não terá somente efeito direto sobre o problema, mas também indireto, reduzindo manifestações de discriminação. 


\section{Colaboradores}

W. Nishida contribuiu na concepção e elaboração do manuscrito, análise e interpretação dos dados, revisão crítica do conteúdo e aprovação do texto final. E. Kupek e C. Zanelatto contribuíram na análise e interpretação dos dados, revisão crítica do conteúdo, aprovação do manuscrito final. J. L. Bastos contribuiu na concepção, coordenação e supervisão da pesquisa, análise e interpretação dos dados, revisão crítica do conteúdo e aprovação do texto final.

\section{Informações adicionais}

ORCID: Waleska Nishida (0000-0002-4937-5124); Emil Kupek (0000-0001-6704-1673); Carla Zanelatto (0000-0002-6168-9898); João Luiz Bastos (0000-0002-1816-0745).

\section{Agradecimentos}

Aos participantes da pesquisa; à Coordenação de Aperfeiçoamento de Pessoal de Nível Superior Brasil (CAPES - Código de Financiamento 001); e ao Conselho Nacional de Desenvolvimento Científico e Tecnológico (CNPq), Ministério da Ciência, Tecnologia, Inovações e Comunicações do Brasil.

\section{Referências}

1. Whelton PK, Carey RM, Aronow WS, Casey Jr. DE, Collins KJ, Dennison Himmelfarb C, et al. 2017 ACC/AHA/AAPA/ABC/ACPM/AGS/ APhA/ASH/ASPC/NMA/PCNA Guideline for the Prevention, Detection, Evaluation, and Management of High Blood Pressure in Adults. Executive summary: a report of the American College of Cardiology/American Heart Association Task Force on Clinical Practice Guidelines. Hypertension 2018; 71:1269-324.

2. Leng B, Jin YN, Li G, Chen L, Jin N. Socioeconomic status and hypertension: a meta-analysis. J Hypertens 2015; 33:221-9.

3. Mills KT, Bundy JD, Kelly TN, Reed JE, Kearney PM, Reynolds K, et al. Global disparities of hypertension prevalence and control: a systematic analysis of population-based studies from 90 countries. Circulation 2016; 134:44150.

4. Picon RV, Fuchs FD, Moreira LB, Riegel G, Fuchs SC. Trends in prevalence of hypertension in Brazil: a systematic review with metaanalysis. PLoS One 2012; 7:e48255.

5. Cipullo JP, Martin JF, Ciorlia LA, Godoy MR, Cacao JC, Loureiro AA, et al. Hypertension prevalence and risk factors in a Brazilian urban population. Arq Bras Cardiol 2010; 94:519-26

6. Alves RF, Faerstein E. Educational inequalities in hypertension: complex patterns in intersections with gender and race in Brazil. Int $\mathrm{J}$ Equity Health 2016; 15:146.

7. Barber S, Diez Roux AV, Cardoso L, Santos $\mathrm{S}$, Toste V, James S, et al. At the intersection of place, race, and health in Brazil: residential segregation and cardio-metabolic risk factors in the Brazilian Longitudinal Study of Adult Health (ELSA-Brasil). Soc Sci Med 2018; 199:67-76.

8. Kaufman JS, Cooper RS. Commentary: considerations for use of racial/ethnic classification in etiologic research. Am J Epidemiol 2001; 154:291-8.

9. Ben-Shlomo Y, Cooper R, Kuh D. The last two decades of life course epidemiology, and its relevance for research on ageing. Int J Epidemiol 2016; 45:973-88.

10. Hallqvist J, Lynch J, Bartley M, Lang T, Blane D. Can we disentangle life course processes of accumulation, critical period and social mobility? An analysis of disadvantaged socio-economic positions and myocardial infarction in the Stockholm Heart Epidemiology Program. Soc Sci Med 2004; 58:1555-62.

11. Leubolt B. Social policies and redistribution in Brazil. Geneva: International Labour Office/ Global Labour University; 2014.

12. Torche F. Intergenerational mobility and inequality: the Latin American case. Annu Rev Sociol 2014; 40:619-42.

13. Mahlmeister R, Ferreira SG, Veloso F, Menezes Filho N, Komatsu BK. Revisitando a mobilidade intergeracional de educação no Brasil. São Paulo: Insper - Instituto de Ensino e Pesquisa; 2017. 
14. Guimarães JMN, Griep RH, Clarke PJ, Fonseca MJM, Barreto SM, Giatti L, et al. Intra-generational social mobility and changes in blood pressure: longitudinal analysis from the ELSABrasil Study. Am J Hypertens 2018; 31:672-8.

15. Marteleto LJ. Educational inequality by race in Brazil, 1982-2007: structural changes and shifts in racial classification. Demography 2012; 49:337-58.

16. Hasenbalg C. Discriminação e desigualdades raciais no Brasil. 2a Ed. Rio de Janeiro: Editora UFMG; 2005.

17. Boing AC, Peres KG, Boing AF, Hallal PC, Silva NN, Peres MA. EpiFloripa Health Survey: the methodological and operational aspects behind the scenes. Rev Bras Epidemiol 2014; $17: 147-62$.

18. Krieger N, Williams DR, Moss NE. Measuring social class in US public health research: concepts, methodologies, and guidelines. Annu Rev Public Health 1997; 18:341-78.

19. Saiba como é a divisão do sistema de educação brasileiro. Agência Brasil 2014. http://www. brasil.gov.br/educacao/2014/05/saiba-como -e-a-divisao-do-sistema-de-educaçãoi-brasi leiro/view (acessado em 30/Jul/2014).

20. Fromme K, Corbin WR, Kruse MI. Behavioral risks during the transition from high school to college. Dev Psychol 2008; 44:1497-504.

21. Organisation for Economic Co-operation and Development. Education at a glance 2011: OECD Indicators. Paris: OECD Publishing; 2011.

22. Janicki-Deverts D, Cohen S, Matthews KA, Jacobs DR, Jr. Sex differences in the association of childhood socioeconomic status with adult blood pressure change: the CARDIA study. Psychosom Med 2012; 74:728-35.

23. Bastos JL, Faerstein E, Celeste RK, Barros AJ. Explicit discrimination and health: development and psychometric properties of an assessment instrument. Rev Saúde Pública 2012; 46:269-78.

24. La Veist TA. Beyond dummy variables and sample selection: what health services researchers ought to know about race as a variable. Health Serv Res 1994; 29:1-16.

25. Sarki AM, Nduka CU, Stranges S, Kandala NB, Uthman OA. Prevalence of hypertension in low- and middle-income countries: a systematic review and meta-analysis. Medicine (Baltimore) 2015; 94:e1959.

26. Instituto Brasileiro de Geografia e Estatística. Pesquisa Nacional de Saúde 2013. Rio de Janeiro: Instituto Brasileiro de Geografia e Estatística; 2014.

27. Agência Nacional de Saúde Suplementar; Ministério da Saúde. Vigitel Brasil 2017 Saúde Suplementar: vigilância de fatores de risco e proteção para doenças crônicas por inquérito telefônico [recurso eletrônico]. Brasília: Ministério da Saúde; 2018.
28. Paradies Y, Ben J, Denson N, Elias A, Priest N, Pieterse A, et al. Racism as a determinant of health: a systematic review and meta-analysis. PLoS One 2015; 10:e0138511.

29. Ordunez P, Kaufman JS, Benet M, Morejon A, Silva LC, Shoham DA, et al. Blacks and Whites in the Cuba have equal prevalence of hypertension: confirmation from a new population survey. BMC Public Health 2013; 13:169.

30. Bowen ME. Coronary heart disease from a life-course approach: findings from the health and retirement study, 1998-2004. J Aging Health 2010; 22:219-41.

31. Rodríguez López S, Bensenor IM, Giatti L, Molina MDC, Lotufo PA. Association between maternal education and blood pressure: mediation evidence through height components in the Brazilian Longitudinal Study of Adult Health (ELSA-Brasil). Ann Hum Biol 2017; 44:243-25

32. Högberg L, Cnattingius S, Lundholm C, Sparen P, Iliadou AN. Intergenerational social mobility and the risk of hypertension. J Epidemiol Community Health 2012; 66:e9.

33. James SA, Van Hoewyk J, Belli RF, Strogatz DS, Williams DR, Raghunathan TE. Life-course socioeconomic position and hypertension in African American men: the Pitt County Study. Am J Public Health 2006; 96:812-7.

34. Cutler DM, Lleras-Muney A. Understanding differences in health behaviors by education. $\mathrm{J}$ Health Econ 2010; 29:1-28.

35. Zimmerman E, Woolf SH. Understanding the relationship between education and health. Washington DC: Institute of Medicine; 2014. (Discussion Paper).

36. Kaiser P, Diez Roux AV, Mujahid M, Carnethon M, Bertoni A, Adar SD, et al. Neighborhood environments and incident hypertension in the multi-ethnic study of atherosclerosis. Am J Epidemiol 2016; 183:988-97.

37. Poulton R, Caspi A, Milne BJ, Thomson WM, Taylor A, Sears MR, et al. Association between children's experience of socioeconomic disadvantage and adult health: a life-course study. Lancet. 2002; 360:1640-5.

38. Krieger N. Embodiment: a conceptual glossary for epidemiology. J Epidemiol Community Health 2005; 59:350-5.

39. Pinheiro L, Fontoura NO, Querino AC, Almeida CR, Firmino CR, Silva FHE, et al. Retrato das desigualdades de gênero e raça. Brasília: Instituto de Pesquisa Econômica Aplicada; 2015. 


\section{Abstract}

Systemic arterial hypertension (SAH) or high blood pressure a serious global public health problem marked by social inequalities. There are few studies on SAH in Brazil with a life-course theoretical perspective. The current article aims to analyze the relationship between intergenerational educational mobility (IEM) and SAH in Brazilian adults, verifying the impact of interpersonal and color/"race" discrimination on this relationship. The authors analyzed data from 1,720 adults (20-59 years) and their parents in the EpiFloripa Adult Study. Random-effects multilevel regression models were estimated. The fixed effects showed an inverse relationship between IEM and odds of $S A H$, with statistical significance for high IEM (paternal model: $O R$ [odds ratio] $=0.39, p=0.006$; maternal model: $O R=0.35, p=0.002$; and family model: $O R=0.35, p=0.001$ ). Meanwhile, interaction models showed that situations of discrimination can act jointly with unfavorable IEM, increasing the odds of SAH, especially among black and brown individuals. The study concludes that persistently high IEM is capable of significantly reducing the odds of $S A H$, while discrimination can intensify the effect of low education, especially in socially marginalized population segments.

Social Inequity; Cardiovascular Diseases; Racism; Social Mobility; Multilevel Analysis

\section{Resumen}

La hipertensión arterial sistémica (HAS) es un problema relevante de salud pública mundial, marcado por desigualdades sociales. En Brasil, los estudios sobre la HAS, adoptando una perspectiva teórica de curso de vida, son escasos. El objetivo de este artículo es analizar la relación entre movilidad educacional intergeneracional (MEI) y HAS en adultos brasileños, verificando el impacto de la discriminación interpersonal y del color/ "raza" en esa relación. Se analizaron datos de los padres y de 1.720 adultos, entre 20 y 59 años, del Estudio EpiFloripa Adulto. Se estimaron modelos de regresión multinivel con efectos aleatorios. Los efectos fijos mostraron una relación inversa entre MEI $y$ odds de HAS, con significancia estadística para MEI alta (modelo paterno: $O R$ [odds ratio] = 0,39, $p=0,006$; modelo materno: $O R=0,35$, $p=0,002 ; y$ modelo familiar: $O R=0,35$, $p=0,001)$. Los análisis de interacción demostraron, a su vez, que situaciones de discriminación pueden actuar conjuntamente con la MEI desfavorable, elevando la odds de HAS, especialmente entre negros y mulatos/mestizos. Se concluye que una MEI constantemente alta es capaz de reducir significativamente la odds de HAS, sin embargo, la discriminación puede intensificar el efecto de bajos niveles de educación, especialmente en segmentos de la población socialmente marginados.

Inequidad Social; Enfermedades Cardiovasculares; Racismo; Movilidad Social; Análisis Multinivel
Recebido em 10/Fev/2019

Versão final reapresentada em 17/Set/2019 Aprovado em 25/Out/2019 